\title{
Low Cost, Lightweight Solar Modules Based on Organic Photovoltaic Technology
}

Konarka Technologies, NREL, University of Delaware

\author{
SAI Final Review Meeting \\ (September, 2010)
}




\title{
Agenda
}

\section{1] Overview}

\author{
2] Stability
}

3] Performance

$\mathbf{N}$-type materials

Bottom grid

Technology Comparison 


\section{Overview - Targets}

Timeline

- Project start date: July 1, 2007

- Project end date: June 30, 2010

- Percent complete: $\mathbf{1 0 0 \%}$

\section{Budget}

- Total project funding: $\$ 8.79 \mathrm{M}$

- Project Spending

- DOE share: \$3.64M (41\%)

- Contractor share: $\$ 5.15 \mathrm{M}$
Barriers

- Stability

- Accelerated aging (1000hrs@85 $\mathrm{C} / 85 \% \mathrm{RH})$

- Rooftop stability (>10yrs)

- Performance

- Cell performance $>\mathbf{7 \%}$

- Module performance 7\%

- Cost

- $\sim \$ 0.5 /$ watt

\section{Partners}

- Project lead: Konarka Technologies

- Interactions/Collaborations

- NREL and The University of Delaware 


\section{Overview - Results}

Objectives (Project Year 3)

In order to produce solar modules for rooftop applications the performance and the lifetime must be improved to $5 \%-7 \%$ and $>10$ year life.

Task 1. Stability

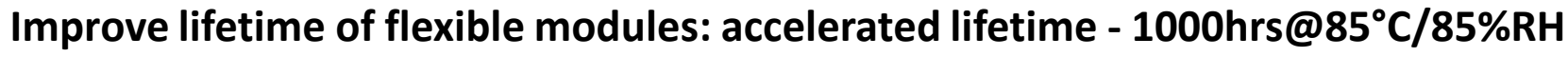

Approaches taken and results:

- Adhesives with filler - advantage demoed (in advanced development at KNB)

- Solution coatable barrier - improvements demoed (more work needed)

- Degradation mechanism - oxygen and water mechanisms demoed

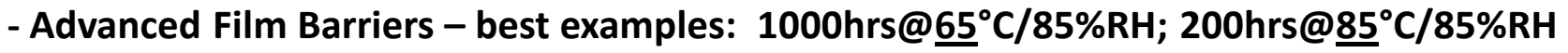

- Glass Packaging - $>2000 \mathrm{hrs} @ 85^{\circ} \mathrm{C} / 85 \%$ RH (passes IEC test: 20 vears)

Task 2. Performance - n-Type materials

Stopped work - year 2

Task 3. Performance - Replace bottom electrode (ITO) with metallic grid

Improve performance of cells and modules by use of a grid: improved \%T and sheet resistance

- Surface roughness - target $5 \mathrm{~nm}$ rms; current: $2 \mathrm{~nm}$ rms

- Optical transmission - target $>85 \% \mathrm{~T}$; current $85-90 \% \mathrm{~T}$

- Sheet resistance - target $50 \mathrm{hms} / \mathrm{sq}$; current $<1 \mathrm{ohm} / \mathrm{sq}$

- Cell performance: $>2.5 \%$ (best), 2.0\% (average) efficiency (P3HT/PCBM active layer; lab cells)

- Coating/printing - screen, flexo and gravure are options - being evaluated 


\section{Stability}




\section{Task 1. Stability}

\section{Target: 1000hrs @ 85C/85\%RH}

\section{Summary}

1] Adhesives / fillers to aid barrier properties - done, in production

2] Solution coatable barriers

- target WVTR $\sim 10^{-3} \mathrm{~g} / \mathrm{m} 2 /$ day

- current state $\sim 10^{-1} \mathrm{~g} / \mathrm{m} 2 /$ day

3] Degradation mechanisms

- oxygen attacks polymer : reversibly and irreversibly

- water affects morphology and interfaces

4] Flexible modules with flexible barriers: $1000 \mathrm{hrs} @ 65^{\circ} \mathrm{C} / 85 \% \mathrm{RH}$

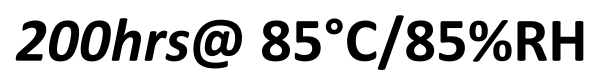

5] Glass packaged flexible modules: >2000hrs @ 85 C/85\%RH 


\section{Module Stability - Effect of Buffer Layer Flexible Barriers}

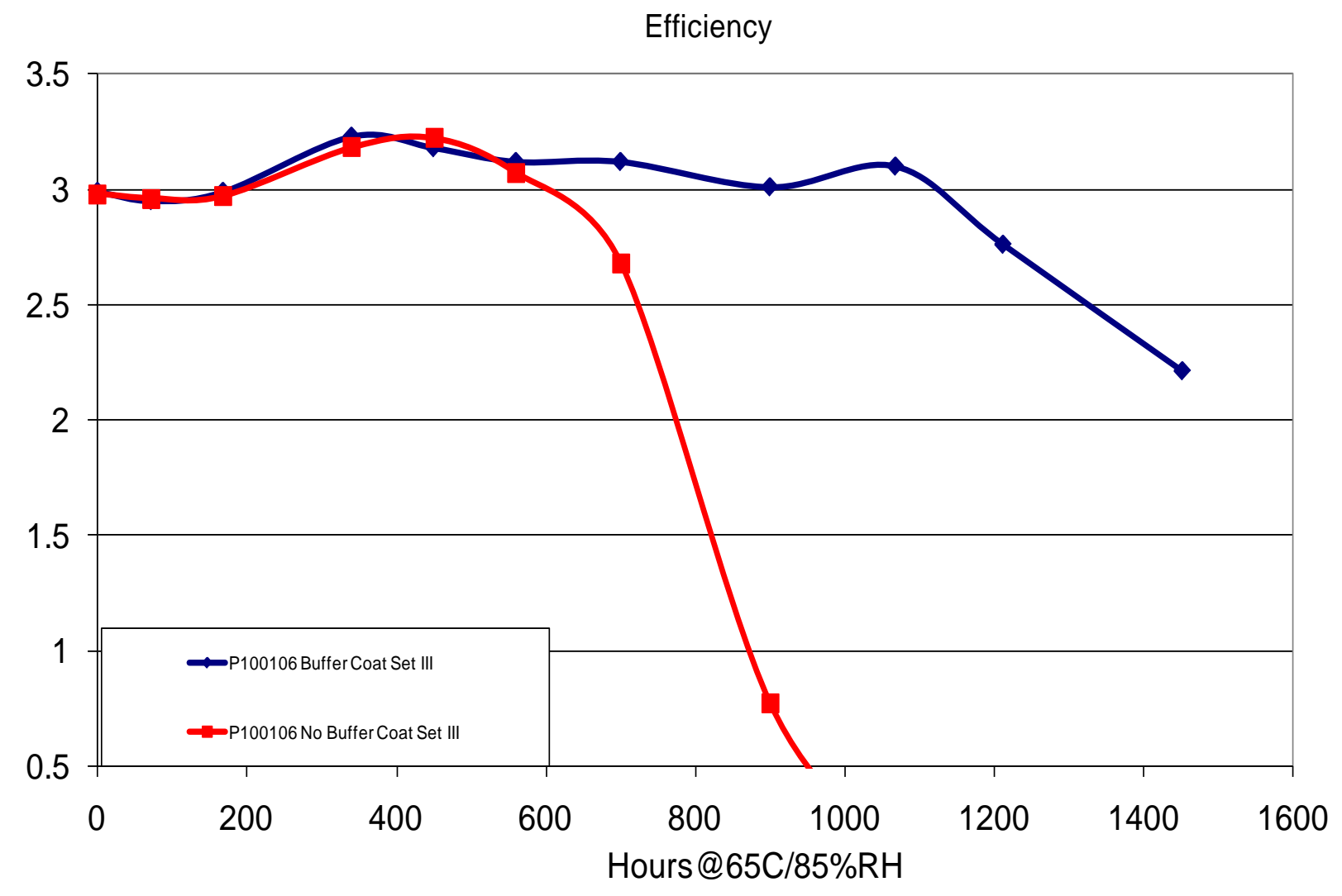

Summary: Buffer layer + filler improves stability accelerated aging 


\section{Rooftop Stability (Lowell, MA) Flexible Barriers}

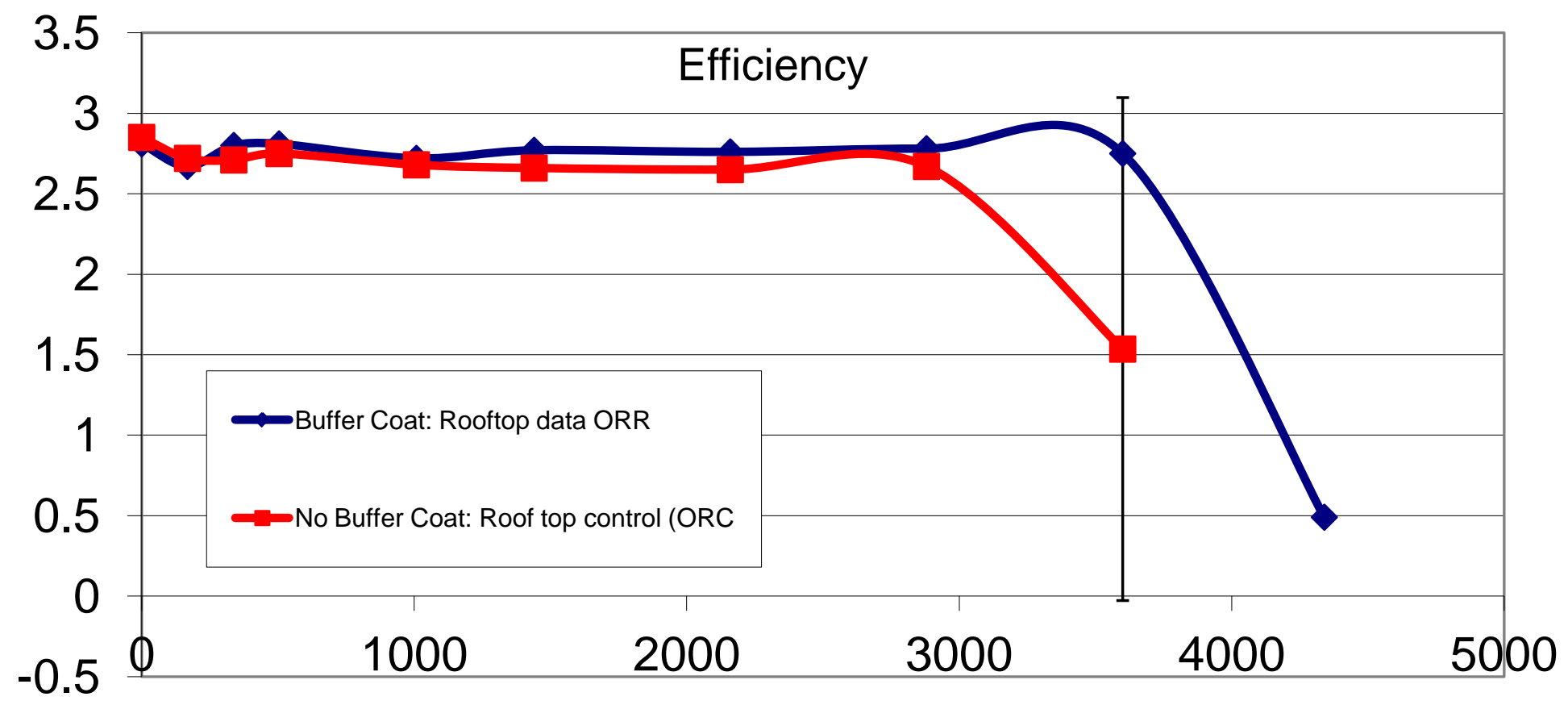

Hours

Summary: Rooftop testing in Lowell , MA shows very good stability 


\section{Module Stability - Glass Packaging}

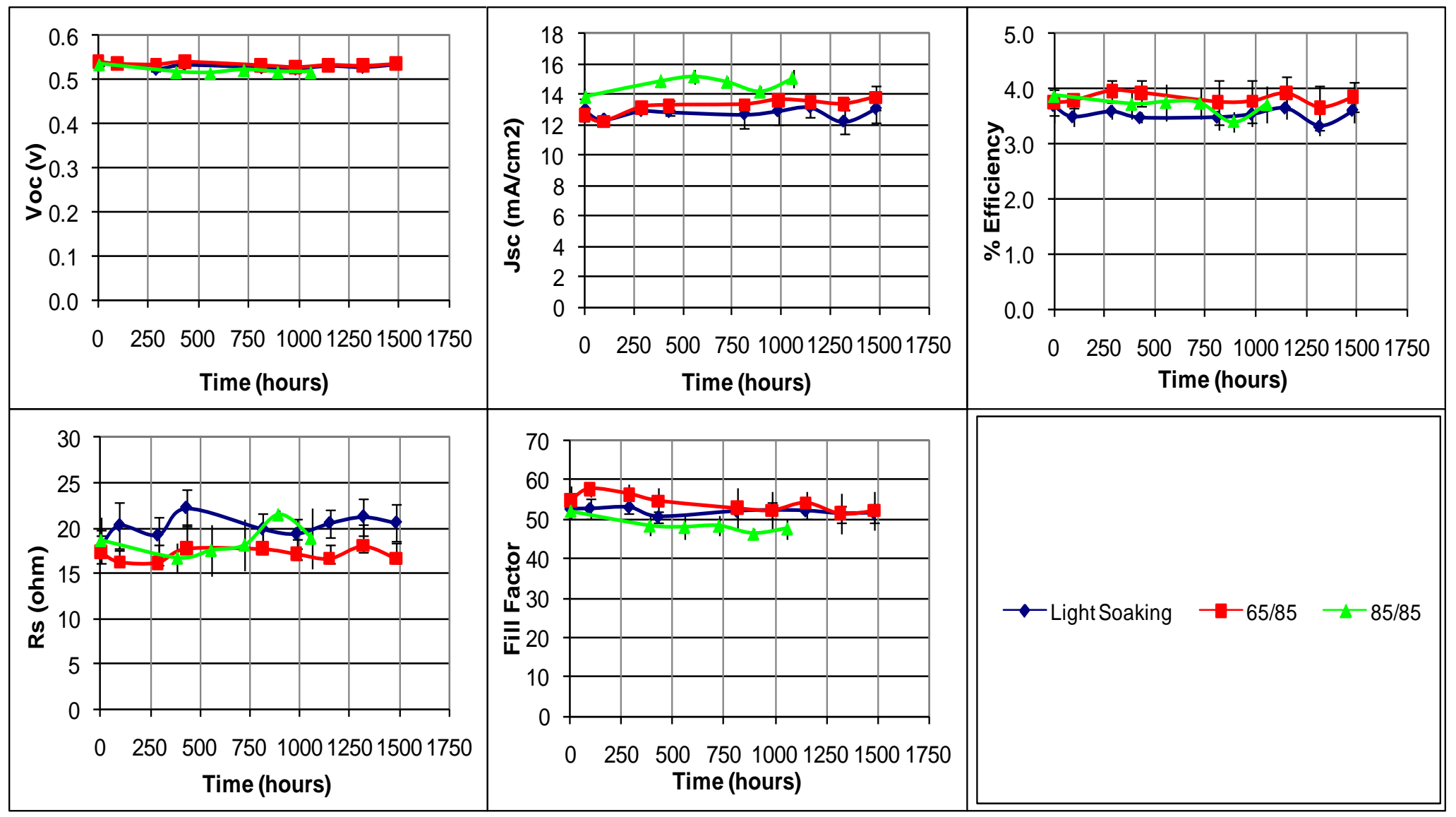

Summary: the polymers in all layers are inherently stable 


\section{Glass Module Stability}

Device ID: F10 0 hours

Jun 08, 2010 14:06

Spectrum: ASTM G173 global

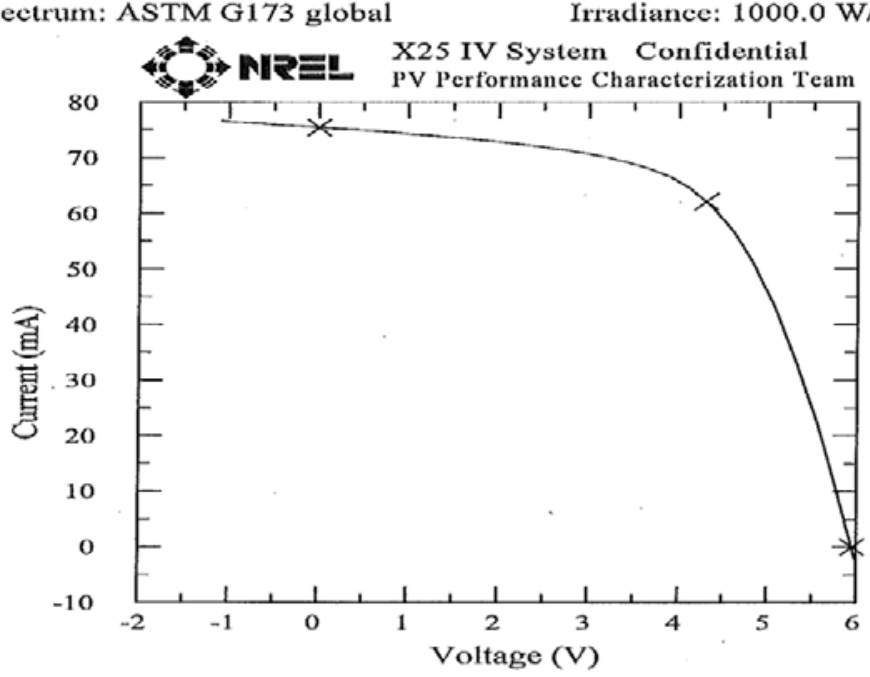

$\mathrm{v}_{\mathrm{oc}}=5.9560 \mathrm{~V}$

$\mathrm{I}_{\mathrm{so}}=75.450 \mathrm{~mA}$

Fill Factor $=59.56 \%$

$\mathrm{I}_{\max }=62.127 \mathrm{~mA}$

$\mathrm{P}_{\max }=0.2676 \mathrm{~W}$
Device Temperature: $25.0 \pm 0.5^{\circ} \mathrm{C}$

Irradiance: $1000.0 \mathrm{~W} / \mathrm{m}^{2}$

\section{0\% Power Reduction \\ @ 85을 $\mathrm{C}$ \% RH (990hrs)}

Device ID: F10 0990 hours

Device Temperature: $24.9 \pm 0.5^{\circ} \mathrm{C}$

Aug 12, 2010 12:44

Spectrum: ASTM G173 global

Irradiance: $1000.0 \mathrm{~W} / \mathrm{m}^{2}$

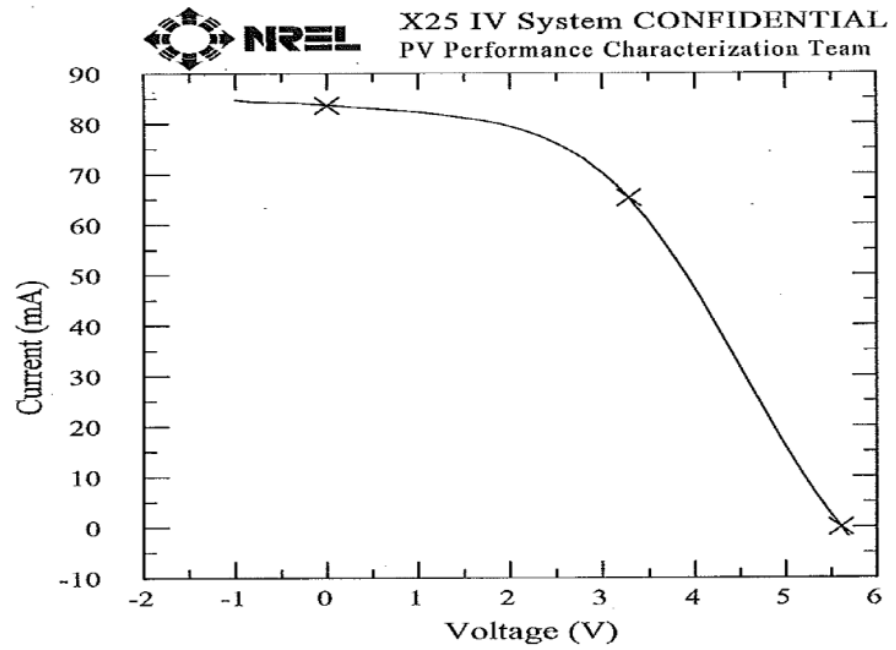

$\mathrm{V}_{\mathrm{oc}}=5.6102 \mathrm{~V}$

$\mathrm{I}_{\mathrm{sc}}=83.566 \mathrm{~mA}$

$I_{\max }=65.228 \mathrm{~mA}$

$\mathrm{V}_{\max }=3.2820 \mathrm{~V}$
$\mathrm{P}_{\max }=0.2141 \mathrm{~W}$

Fill Factor $=45.66 \%$ 


\section{Module Stability - Hot /Cold Cycling Glass Packaging}
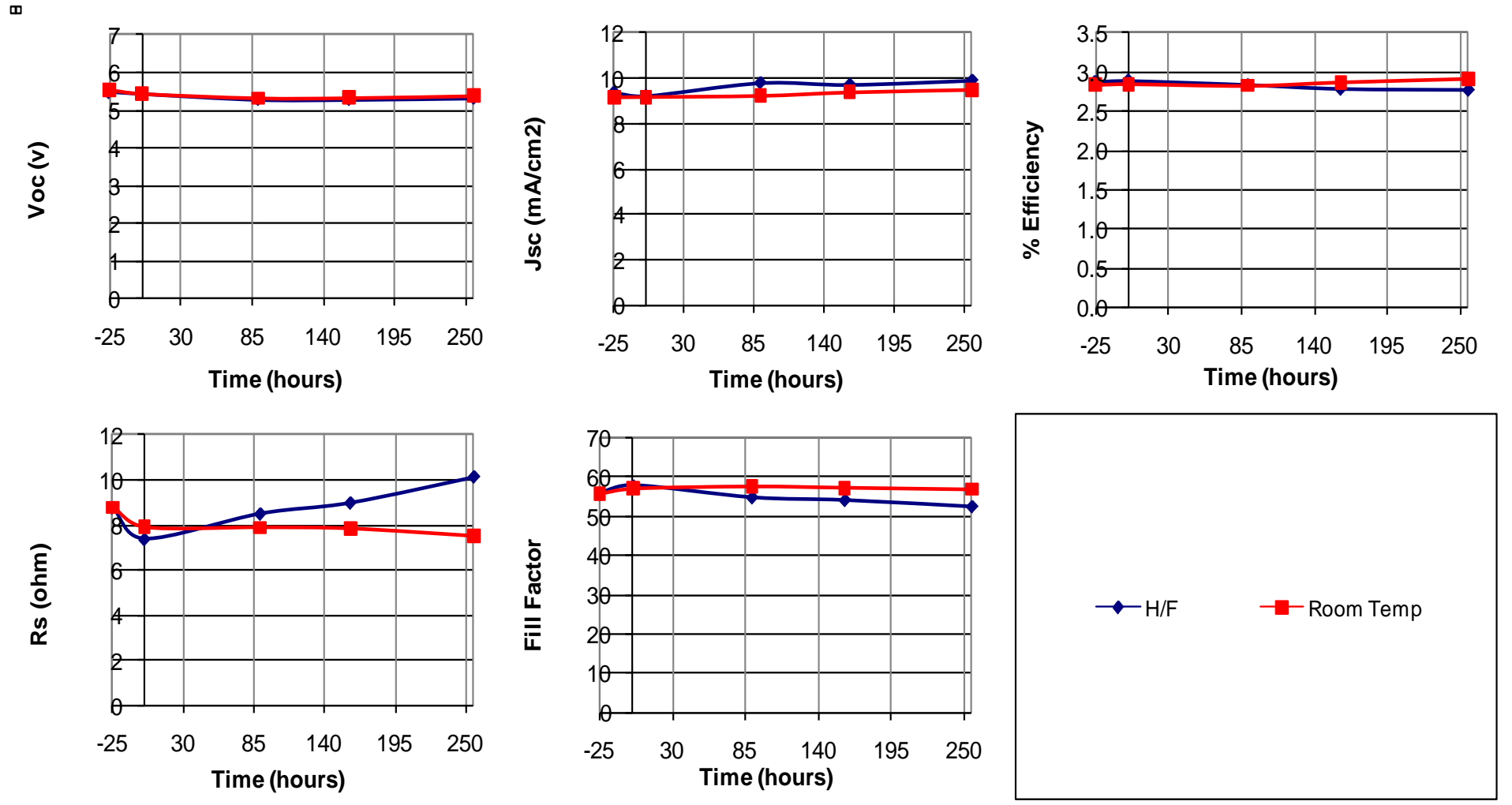

Summary: Hot/cold cycling has no effect on stability - $<5 \%$ decrease in Efficiency after 250 hours 


\section{Ratio of final to initial parameters for flexible modules in Dry Heat $\left(65^{\circ} \mathrm{C} / 10 \% \mathrm{RH}\right)$}

\begin{tabular}{|c|c|c|c|c|c|}
\hline Sample ID & Bias & Voc & Isc & FF & Power \\
\hline 230 & $\mathrm{SC}$ & $100.2 \%$ & $101.8 \%$ & $97.3 \%$ & $99.2 \%$ \\
\hline 231 & SC & $101.1 \%$ & $102.0 \%$ & $101.5 \%$ & $104.7 \%$ \\
\hline 219 & MP & $98.3 \%$ & $102.8 \%$ & $97.7 \%$ & $98.8 \%$ \\
\hline 222 & MP & $98.9 \%$ & $102.2 \%$ & $89.8 \%$ & $90.7 \%$ \\
\hline 220 & $\mathrm{OC}$ & $99.3 \%$ & $101.2 \%$ & $95.1 \%$ & $95.6 \%$ \\
\hline 229 & OC & $100.4 \%$ & $100.6 \%$ & $105.7 \%$ & $106.9 \%$ \\
\hline 232 & Dark & $96.6 \%$ & $102.5 \%$ & $95.2 \%$ & $94.2 \%$ \\
\hline 248 & Control & $99.5 \%$ & $101.5 \%$ & $99.3 \%$ & $100.0 \%$ \\
\hline 249 & Control & $99.3 \%$ & $101.3 \%$ & $97.0 \%$ & $97.7 \%$ \\
\hline
\end{tabular}

Summary: Modules are thermally stable (dry heat) 


\section{Ratio of final to initial parameters for flexible modules in Damp Heat $\left(65^{\circ} \mathrm{C} / 85 \%\right)$}

\begin{tabular}{|c|c|c|c|c|c|}
\hline Sample ID & Bias & Voc & Isc & FF & Power \\
\hline 233 & $\mathrm{SC}$ & $96.4 \%$ & $97.2 \%$ & $68.0 \%$ & $63.8 \%$ \\
\hline 234 & SC & $95.1 \%$ & $99.1 \%$ & $64.6 \%$ & $60.9 \%$ \\
\hline 237 & $\mathrm{SC}$ & $97.6 \%$ & $90.5 \%$ & $47.0 \%$ & $41.6 \%$ \\
\hline 238 & MP & $97.7 \%$ & $101.5 \%$ & $69.6 \%$ & $69.2 \%$ \\
\hline 239 & MP & $101.1 \%$ & $100.9 \%$ & $76.8 \%$ & $78.5 \%$ \\
\hline 242 & $\mathrm{MP}$ & $99.6 \%$ & $103.7 \%$ & $79.8 \%$ & $82.5 \%$ \\
\hline 241 & $\mathrm{OC}$ & 101.9\% & $100.9 \%$ & $82.4 \%$ & $84.4 \%$ \\
\hline 244 & $\mathrm{OC}$ & $99.9 \%$ & 103.3\% & $84.2 \%$ & $87.0 \%$ \\
\hline 245 & $\mathrm{OC}$ & $98.8 \%$ & $100.3 \%$ & $78.1 \%$ & $77.6 \%$ \\
\hline 246 & Dark & $94.5 \%$ & $104.6 \%$ & $80.7 \%$ & $79.9 \%$ \\
\hline 248 & Control & $99.5 \%$ & $101.5 \%$ & $99.3 \%$ & $100.0 \%$ \\
\hline 249 & Control & $99.3 \%$ & $101.3 \%$ & $97.0 \%$ & $97.7 \%$ \\
\hline
\end{tabular}

Summary: heat and humidity causes major instability at short circuit 
Solution Coatable Barriers - NREL 



\section{Performance}




\section{Task 2: Performance - n-Type Materials}

\section{Target : 7\% module efficiency}

Summary:

1] n-type polymers difficult to synthesize - stopped

2] n-type small molecules (fullerenes)

30 - 40 synthesized and tested

Deep LUMO allows electron transfer from polymers with deep LUMOs

High cell voltage and improved performance not yet realized due to morphology issues

3] improved cell efficiency with this approach not realized 


\section{Task 3. Performance - Bottom Grids}

Target: Replace ITO with a metallic grid

\section{Summary}

1] a metallic grid is used as the bottom electrode (cell)

surface roughness $<\sim 5 n m$; current value $<\sim 2 n m$

fill layer level with top of grid - done

sheet resistance $<50 \mathrm{hms} / \mathrm{sq}$; current value $<10 \mathrm{hm} / \mathrm{sq}$

$\% \mathrm{~T}>85 \%$; current value $85-90 \% \mathrm{~T}$

2] metallic grid in a module:

targeting equivalent performance to ITO

current status: $2 \%$ efficiency (av.); $2.6 \%$ (best) 


\section{Printed Silver Grid}

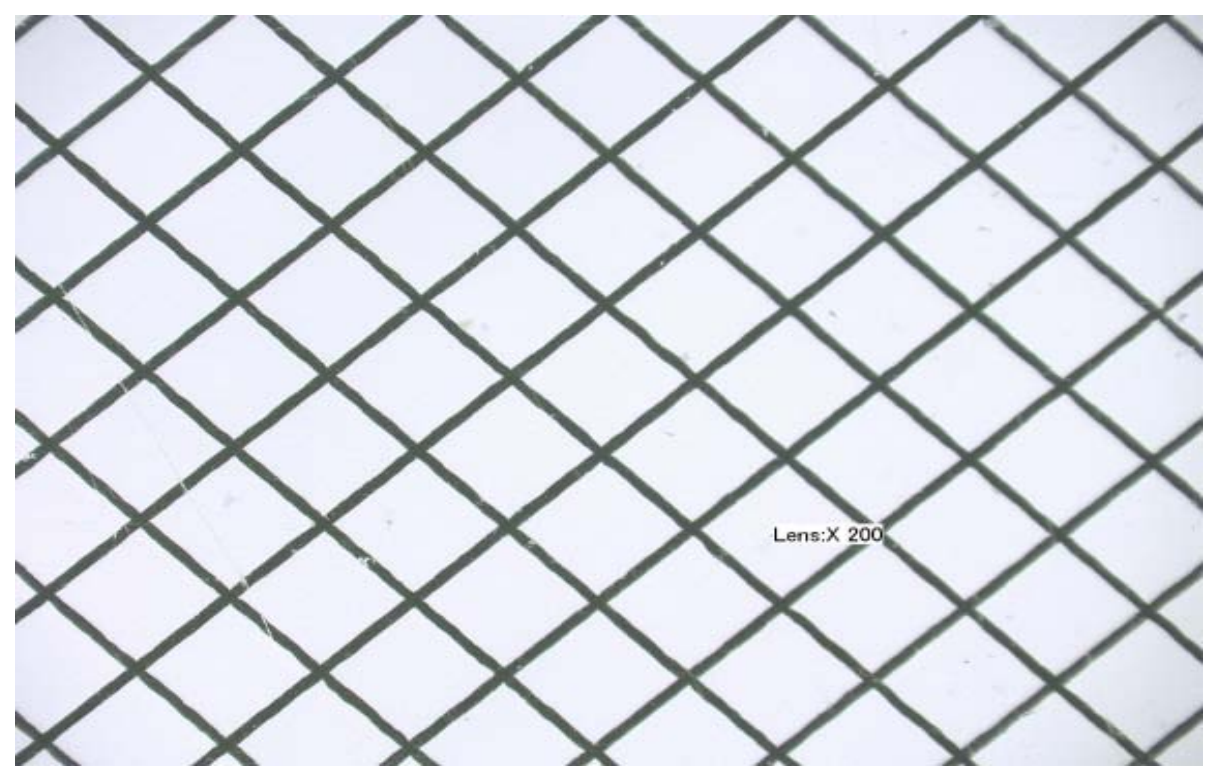

\section{Data}

$\leq 90 \%$ open area

40 - 50um wire width

Sheet resistance $<10 \mathrm{hm} / \mathrm{sq}$

Transmission: $>85 \% \mathrm{~T}$

Stability (no change in conductivity)

$>1000 \mathrm{hrs} @ 65^{\circ} \mathrm{C} / 85 \% \mathrm{RH}$

$>1000 \mathrm{hrs} @ 85^{\circ} \mathrm{C}$ (dry)

Summary: several printing processes can be used to print grids with $40-50$ um wires and spacing between wires will be adjusted to give the desired transmission 


\section{Surface Roughness (AFM) of Grid and Fill layer}

\begin{tabular}{|c|c|c|c|c|}
\hline & Max peak (nm) & $\begin{array}{c}\text { Ten point } \\
\text { height (nm) }\end{array}$ & Av. Peak (nm) & $\begin{array}{c}\text { Surface roughness - } \\
\text { RMS (nm) }\end{array}$ \\
\hline Bare ITO & $138+/-19$ & $68+/-9$ & $21+/-3$ & $\mathbf{6 + / - 1}$ \\
\hline Polyester Substrate & $84+/-3$ & $40+/-2$ & $15+/-7$ & $2+/-\mathbf{0}$ \\
\hline $\begin{array}{c}\text { Surface of Silver grid wires } \\
\text { Surface of the fill between } \\
\text { the grid lines }\end{array}$ & $27+/-8$ & $14+/-6$ & $15+/-9$ & $2+/-1$ \\
\hline
\end{tabular}

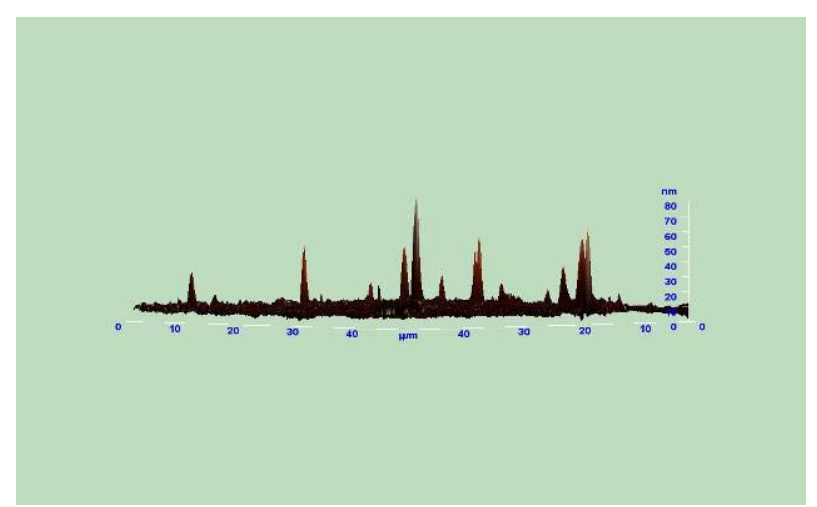

Polyester Substrate

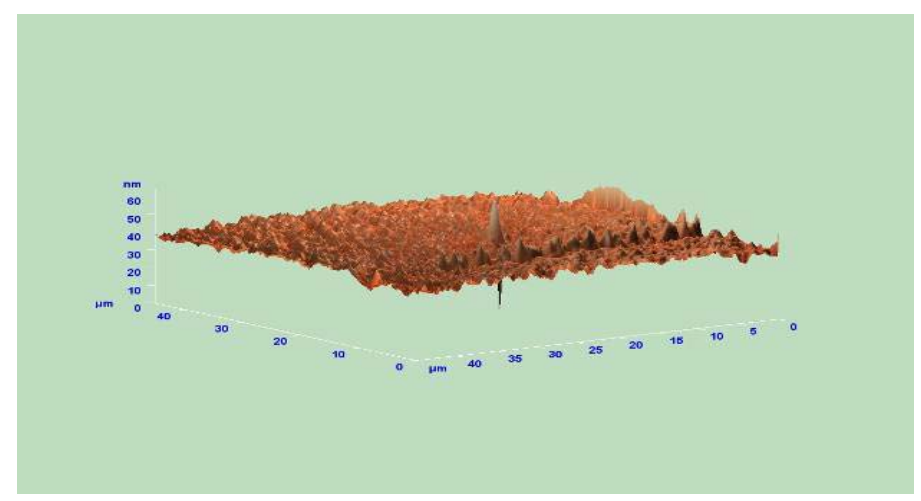

Space Between Grid Wires

Summary: extremely low surface roughness of the bottom grid is a key coating a cell without shorts 


\section{Standard Cell Efficiency}

\section{Konarka Technologies \\ organic Cell}

Device ID: TY100716-32

Aug 18, 2010 14:38

Device Temperature: $30.0 \pm 10.0{ }^{\circ} \mathrm{C}$

Spectrum: ASTM G173 global

Device Area: $0.9950 \mathrm{~cm}^{2}$

Irradiance: $1000.0 \mathrm{~W} / \mathrm{m}^{2}$

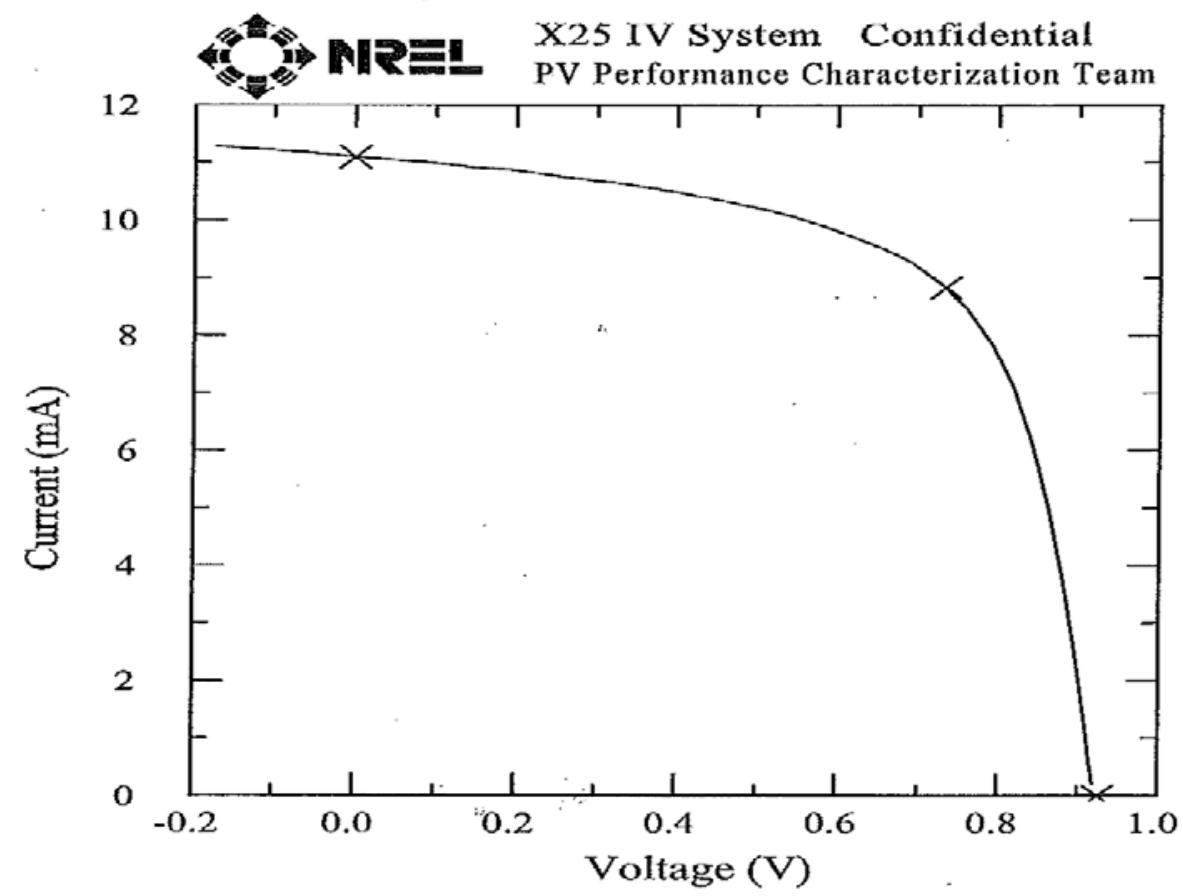

$\mathrm{V}_{\mathrm{oc}}=0.9259 \mathrm{~V}$

$\mathrm{I}_{\mathrm{sc}}=11.093 \mathrm{~mA}$

$J_{\mathrm{sc}}=11.149 \mathrm{~mA} / \mathrm{cm}^{2}$

$I_{\max }=8.8259 \mathrm{~mA}$

Fill Factor $=63.09 \%$

$\mathrm{V}_{\max }=0.7342 \mathrm{~V}$

$\mathrm{P}_{\max }=6.4800 \mathrm{~mW}$

Efficiency $=6.51 \%$

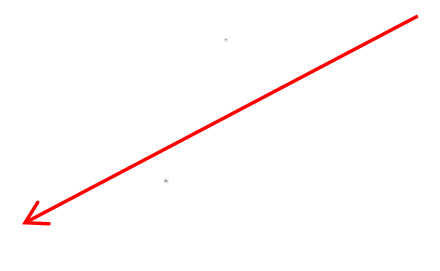




\section{Power Comparison}




\section{SAI Presentation \\ Competitive Performance}

\section{Application:}

Curtain Wall 
Competitive Testing - Energy Collection on 08/01/10

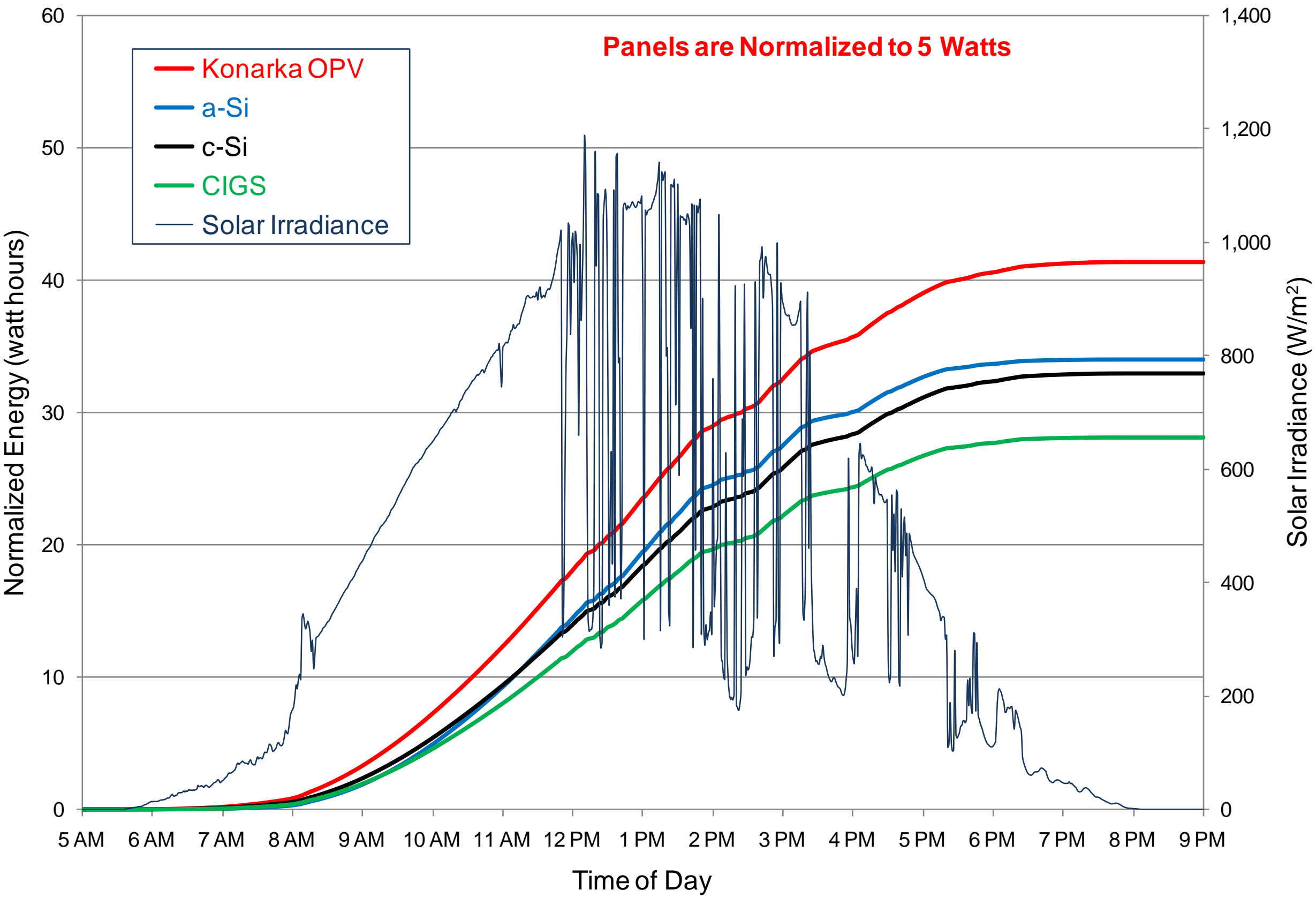




\section{Daily Solar Energy Collection for 08/01/10 - Lowell, MA}

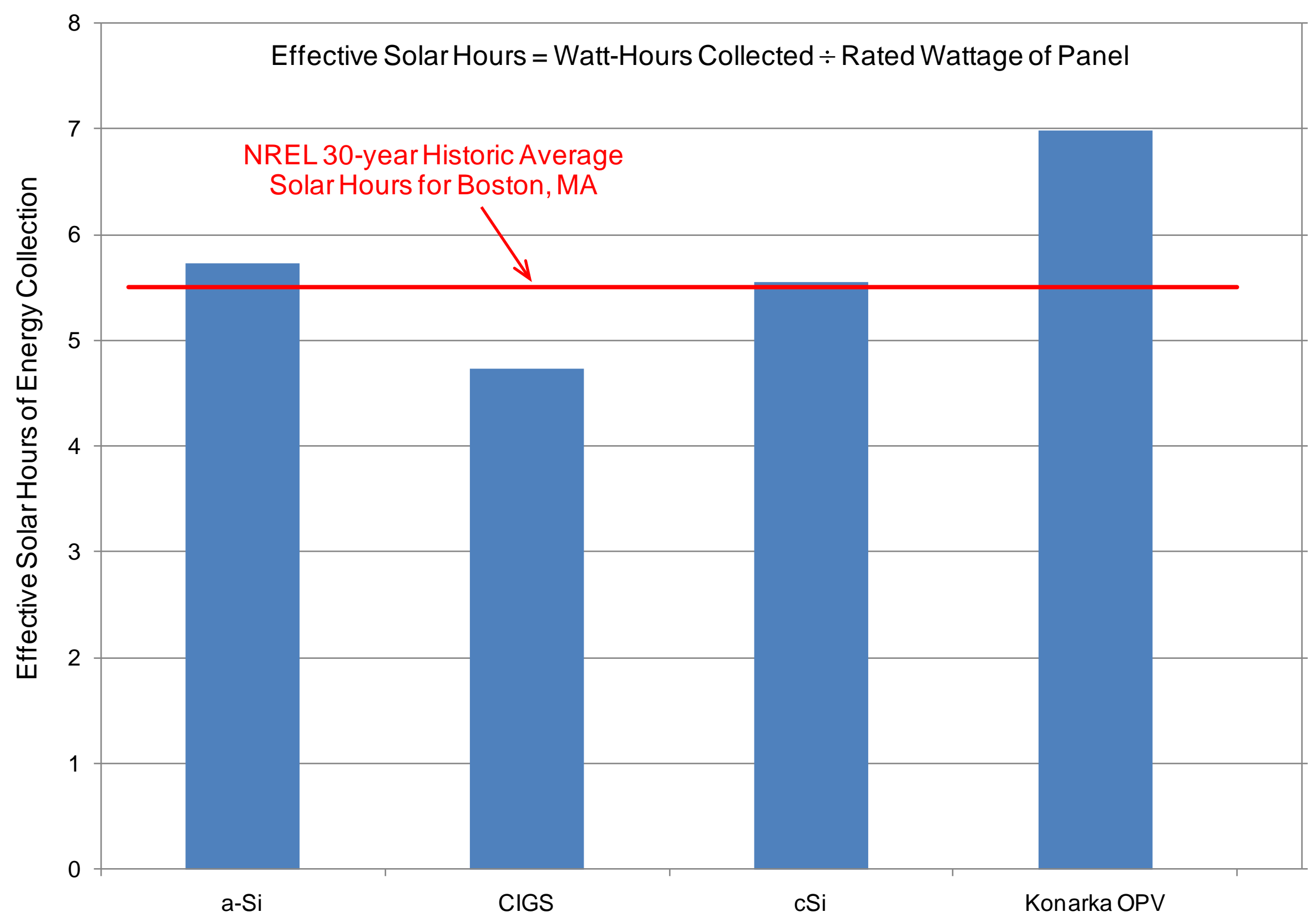




\section{Arch Rooftop Panels (horizontal orientation): Energy Collection}

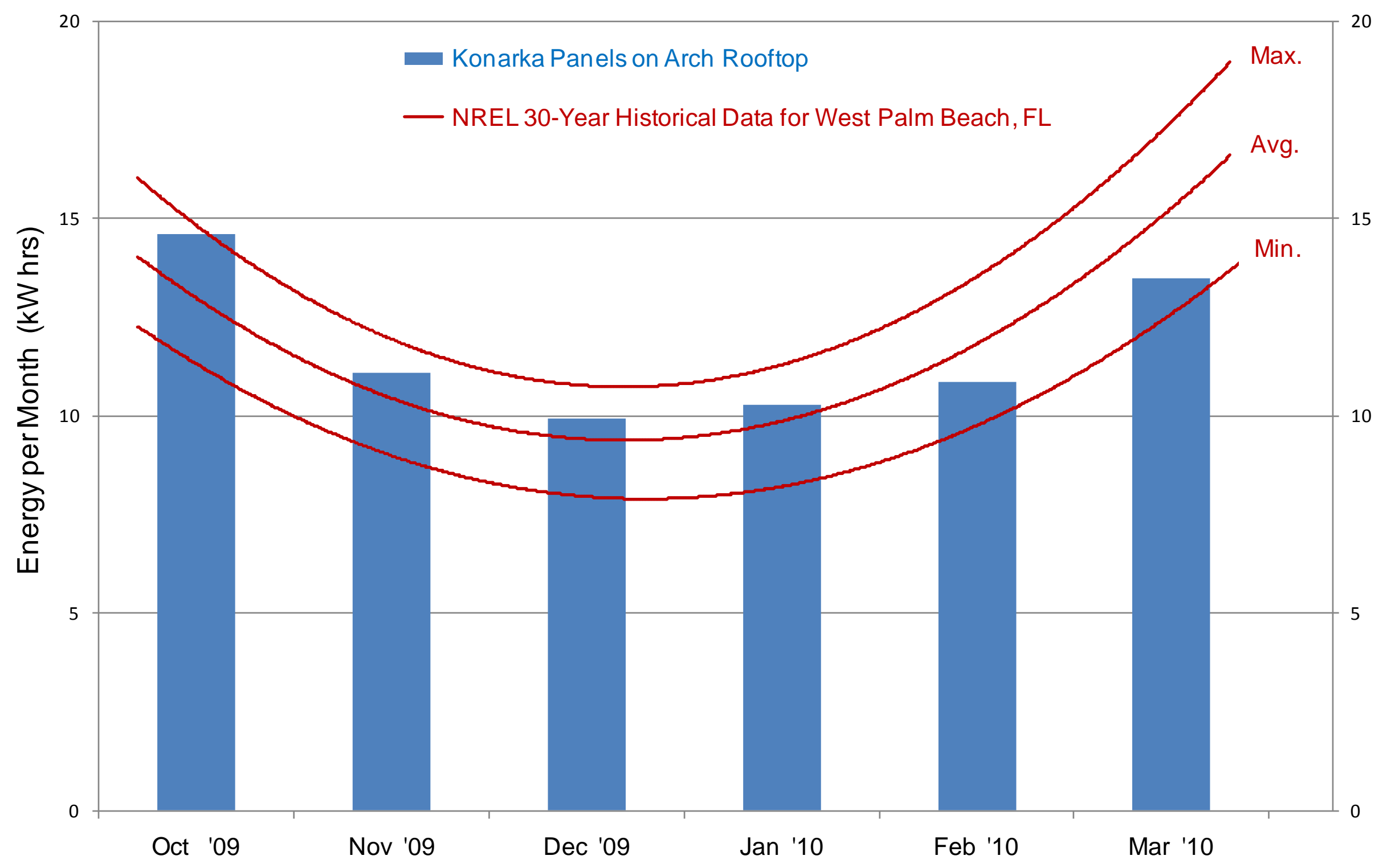




\section{Energy Collection for Different Technologies}

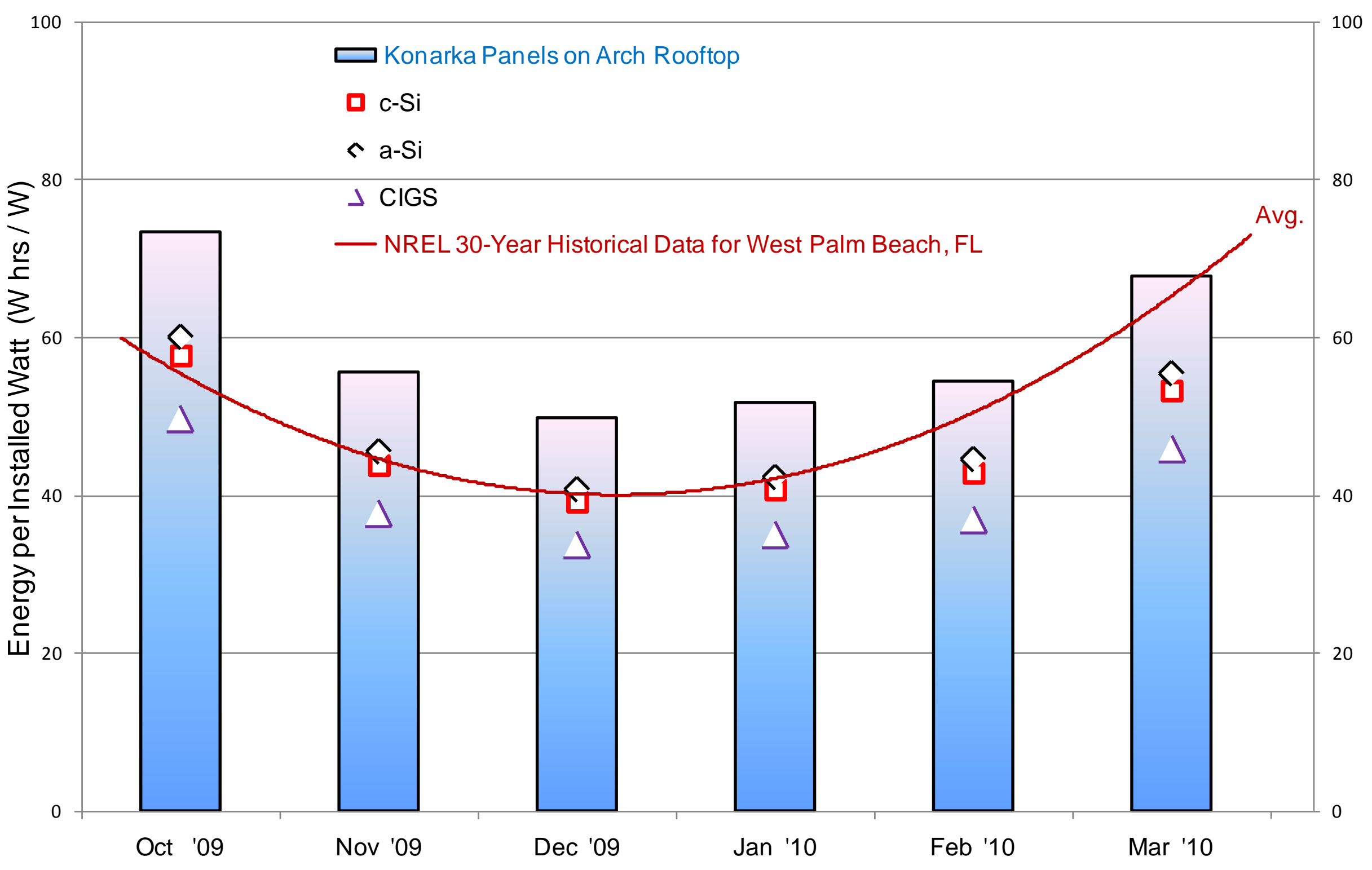


South Curtain Wall Panels: Energy Collection

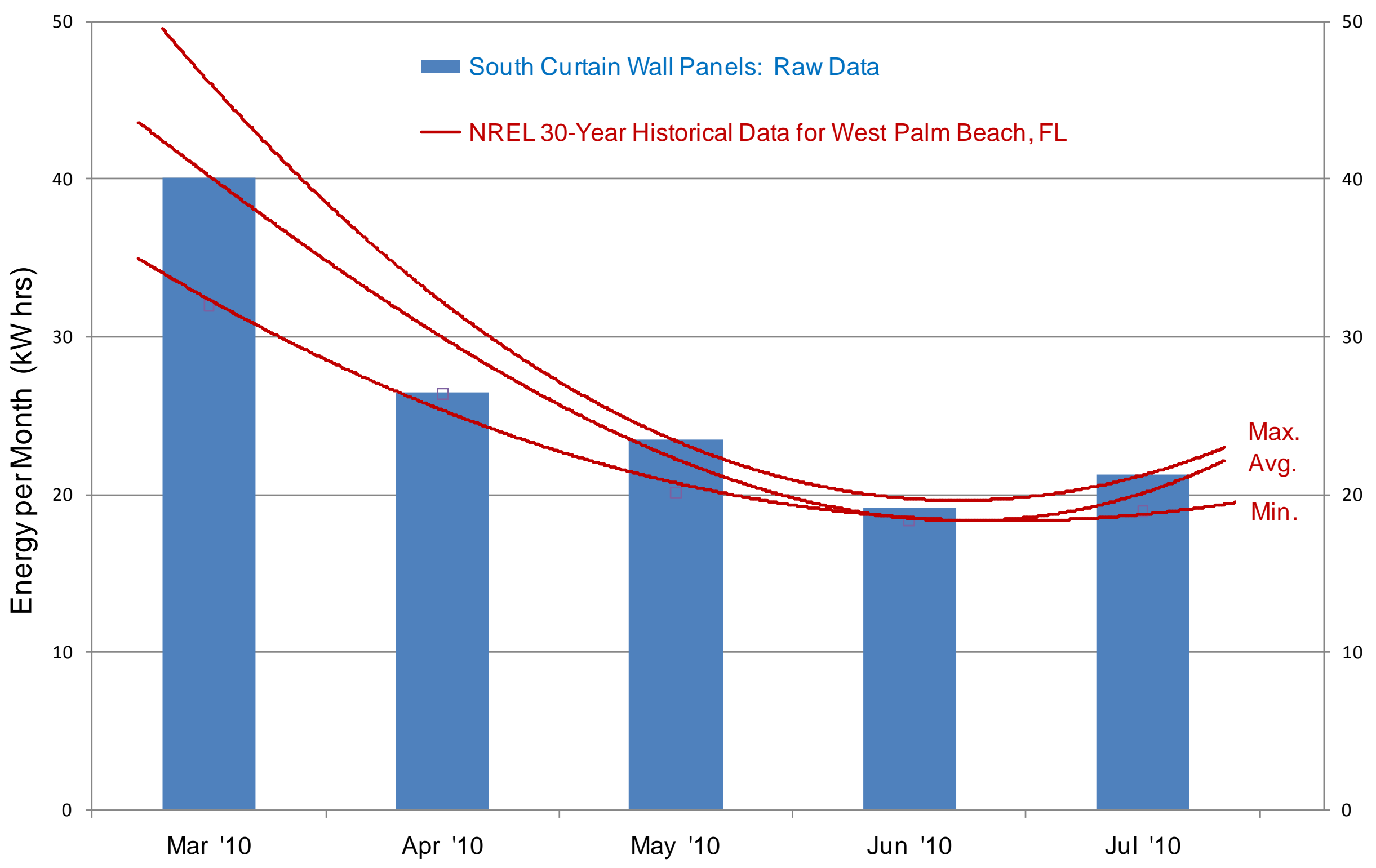




\section{Arch Curtain Wall}

\section{Energy Production by Inverter}

for East \& South Walls

$3 / 01 / 10$ - 7/31/10

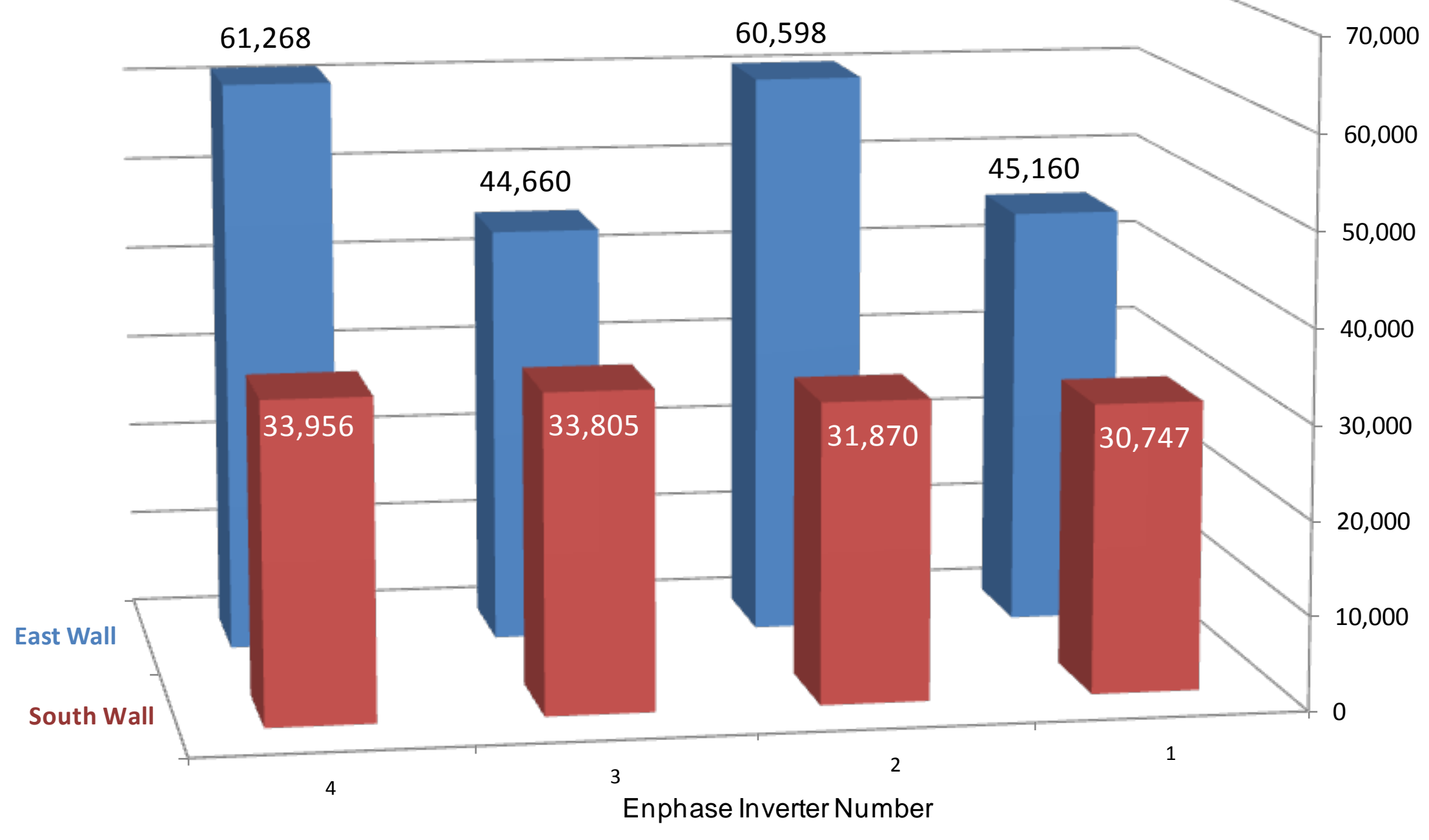




\section{Arch Curtain Wall}

Energy Production by Month

for East \& South Curtain Walls

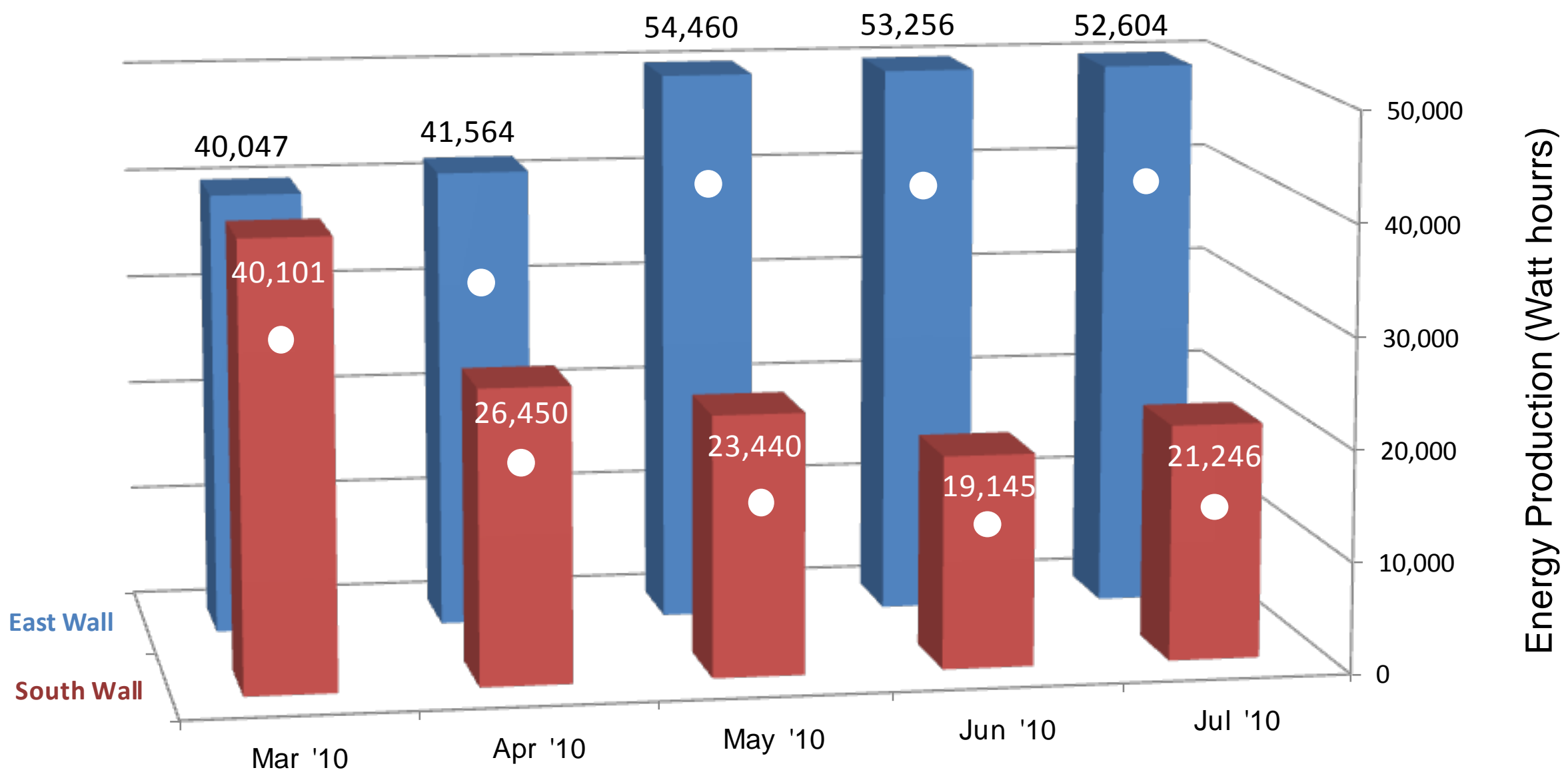

Data for C-Si

Note: the East Wall is Scaled for 16 Panels 


\section{Task 1 - Stability}

- $\quad$ Flexible modules are stable to $1000 \mathrm{hrs}$ at $65^{\circ} \mathrm{C} / 85 \% \mathrm{RH}$

- $\quad$ Flexible modules in glass are stable to $>2000 \mathrm{hrs}$ at $85^{\circ} \mathrm{C} / 85 \% \mathrm{RH}$ (no decrease in performance)

- Adhesive + filler helps stabilize modules

- $\quad$ Solution coatable barriers exhibit good WVTR; work in-progress

\section{Task 2 - Performance: $n$-type charge carriers}

- $\quad \mathrm{N}$-type polymers could not be synthesized

- More than $\mathbf{3 0}$ fullerene derivatives synthesized and tested

- Several deep LUMO derivatives accept charge from deep LUMO polymers: higher voltage observed

- Improvement in cell efficiency not observed - morphology problem

\section{Task 3 - Performance: grid electrode}

- $\quad$ Exceeded flatness and roughness goals

- Exceeds sheet resistance goals

- $\quad$ Achieved \%T goals

- $\quad$ Performance equivalent to ITO - 2\% Efficiency ( av.); work in-progress

\section{Current Cell and Module Performance}

- World record $\left(1 \mathrm{~cm}^{2}\right.$ cell area $)=6.51 \%$

- Cumulative power production larger than competitive technologies 